\title{
Perfil dos idosos com depressão em comunidade do município de Fortaleza
}

\section{Profile of depressed aged in a city community of Fortaleza}

Carina Barbosa Bandeira ${ }^{1}$

\section{Resumo}

A depressão em pacientes idosos tem se tornado problema comum, interferindo na sua qualidade de vida. Este problema de saúde tem sido bastante estudado, pois tem-se percebido a relação entre depressão e idade avançada e uma forma singular de depressão nos idosos. Este trabalho teve como objetivo identificar a presença de depressão nos pacientes idosos da comunidade atendida na área do Curió atendida na Unidade Básica de Saúde da Família Terezinha Parente, no município de Fortaleza, e avaliar a associação entre a doença e o perfil socioeconômico e demográfico da população estudada. Trata-se de um estudo exploratório-descritivo com enfoque quantitativo. Os dados foram coletados por meio de questionário estruturado e da Escala de Depressão Geriátrica (EDG) com 15 itens aplicados em todos os pacientes idosos que aceitaram participar do estudo por agentes comunitários de saúde, os quais foram devidamente treinados para aplicá-los. A prevalência de depressão no presente estudo foi de 37,6\% e a distribuição de casos entre os sexos foi de 84,4\% para mulheres e 15,6\% para homens na população estudada. A prevalência de depressão foi maior nos idosos mais jovens: 59,38\% dos casos entre 60 e 69 anos, 37,5\% entre 70 e 79 anos e 3,12\% acima de 80 anos. Em relação à situação financeira dos idosos do presente estudo, verificou-se que apenas 9,4\% dos idosos com depressão viviam com renda superior a dois salários-mínimos. Observou-se que 74,2\% dos casos de depressão detectados nos idosos da amostra total moram em domicílios multigeracionais, ou seja, com filhos e netos. Dentre idosos com hipertensão ou diabetes, que são casos de depressão segundo a EDG, 10\% apresentam diabetes mellitus, 55\% apresentam hipertensão arterial sistêmica e 35\% apresentam ambas as doenças. Com base no estudo realizado com idosos do bairro Curió, pode-se propor várias ações de promoção de saúde e prevenção de doenças para melhorar a qualidade de vida do idoso atendido nas Unidades Básicas de Saúde da Família do município de Fortaleza.

Palavras-chave: Depressão; Saúde Mental;

Saúde do Idoso.
Key Words: Depression; Mental Health;

Health of the Elderly

${ }^{1}$ Especialista em MFC e Geriatria; Professora Auxiliar, Curso de Medicina, Universidade de Fortaleza, Fortaleza, Ceará, Brasil. 


\begin{abstract}
The depression in senior patients is a very common problem, interfering in their life quality There are many studies considering this health problem, as it has been noticed the relationship between depression and advanced age and a singular kind of depression in seniors. This study-intended to identify cases of depression presence in seniorpatients assisted in the area of Curio Community in the Terezinha Parentein the city of Fortaleza and to evaluate the association between the disease and the socioeconomic and demographic profile of the studied population. It concerns an exploratory-descriptive study with quantitative focus. The data was collected through structured questionnaire and the Scale of Geriatric Depression (SGD) within 15 items which had been applied into in all volunteered patients above 60 years by the community agents which were properly trained to apply it. The prevalence of depression in the present study was of 37,6\% and the distribution of cases between the genders was of $84,4 \%$ affecting for women and $15.6 \%$ affecting for men in the studied population. The prevalence of depression was bigger in aged the youngest ones with 59,38\% of the cases between 60 and 69 years, $37.5 \%$ between 70 and 79 years and $3.12 \%$ above 80 years. In relation to the financial situation of the aged ones of the present study, it was verified that only $9.4 \%$ of the aged ones with depression lived with superior income the 2 minimumwage. It was observed that $74.2 \%$ of the detected cases of depression in the aged ones of the total sample live in multi generation environment, which means, seniors with children and grandchildren. Amongst aged with hipertension or diabetes, which are cases of depression according to SGD, 10\% presents mellitus diabetes, 55\% present hipertension and 35\% present both the illnessess. Based in the study carried through in the aged ones of the Curio, it can be considered that some actions of health promotion and prevention of illnesses to improve the taken care of life quality quality of life of the aged one in the Basic Units of Health of the Family in the City of Fortaleza.
\end{abstract}

\section{Introdução}

Idoso, de acordo com a Organização Mundial de Saúde, inclui as pessoas com idade superior a 60 anos para países em desenvolvimento e 65 anos para países desenvolvidos.
Atualmente, o envelhecimento populacional é um fenômeno global, tanto nos países desenvolvidos como nos em desenvolvimento. Entretanto, os mecanismos que levam a tal envelhecimento são diferentes entre essas nações.

$\mathrm{Na}$ Europa, por exemplo, o aumento na expectativa de vida ao nascimento já havia sido substancial à época em que ocorreram importantes conquistas do conhecimento médico, em meados deste século. Este fato pode ser ilustrado pelo clássico exemplo da redução da mortalidade por tuberculose. A isso se associa a elevação do nível da população, traduzido pela urbanização adequada das cidades, melhoria nutricional, elevação dos níveis de higiene pessoal, melhores condições sanitárias em geral e, particularmente, condições ambientais no trabalho e nas residências muito melhores que antes. Em países em desenvolvimento, por outro lado, aumento substancial na expectativa de vida ao nascimento pode ser observado a partir de $1960^{1}$.

Os países em desenvolvimento vêm apresentando, nas últimas décadas, um progressivo declínio nas suas taxas de mortalidade e, mais recentemente, também nas suas taxas de fecundidade ${ }^{2}$. No Brasil, o aumento da população idosa será da ordem de 15 vezes, entre 1950 e 2025, enquanto o da população como um todo será de não mais que cinco vezes no mesmo período, o que colocará o Brasil como sexto lugar em população senil no mundo.

Atualmente, os idosos do Brasil representam 8,6\% da população, o que equivale ao contingente de 14,5 milhões de pessoas. Em relação a 1991, houve um crescimento de $35,5 \%$ na quantidade de pessoas idosas ${ }^{3}$.

Este aumento abrupto da população idosa no Brasil deveu-se aos avanços na área da saúde, investimentos em educação, saneamento e, também, ao aumento da escolaridade feminina, com consequente crescimento da participação no mercado de trabalho e uso disseminado de métodos contraceptivos.

Uma característica importante da transição demográfica no Brasil foi a feminização da população de gerontes, pois, de 54\% em 1991, passou para 55,1\% em 2000. Apesar de ser um fenômeno universal, no Brasil está 
mais acentuado, visto que a expectativa média de vida das mulheres é oito anos maior que a dos homens, segundo o Censo Demográfico de $2000^{4}$.

A epidemiologia do envelhecimento é uma área emergente, que se ocupa das condições que levam à incapacidade funcional e a subsequente aumento da dependência, além das doenças responsáveis pela morbimortalidade entre idosos ${ }^{5}$. A transição demográfica que vem ocorrendo no Brasil tem provocado diversas mudanças no perfil de saúde neste país, pois as doenças crônico-degenerativas vêm tomando o lugar das doenças infectocontagiosas como primeira causa de mortalidade. Com isso, vários estudos têm sido realizados para detectar fatores de risco para tais doenças a fim de que ações de promoção de saúde e prevenção de doenças possam ser realizadas.

As principais causas definidas de mortalidade entre idosos brasileiros são as doenças do aparelho circulatório, as neoplasias e as doenças do aparelho respiratório, correspondendo a cerca de $60 \%$ do total de óbitos entre homens e mulheres.

Na população envelhecida, a depressão encontrase entre as doenças crônicas mais frequentes que prejudicam a independência funcional do idoso, tornando-se um problema grave de saúde pública, uma vez que há prejuízo na qualidade de vida e aumento nos gastos com serviços de saúde, além de, na maioria das vezes, ser este um problema que envolve os familiares. A associação entre depressão e idade avançada tem despertado vários estudos, tendo sido observado que esta doença difere em suas características quando comparada a outras faixas etárias. Os argumentos que sustentam a tese de que a depressão no idoso é diferente está baseada nas diferenças de sintomatologia. Nos idosos, por exemplo, a depressão se apresenta com sintomas somáticos mais frequentemente com menos história familiar associada e pior resposta ao tratamento clínico. Na verdade, parece que o que diferencia não é a depressão em si, mas, sim as circunstâncias próprias de cada faixa etária.

Entre as principais variáveis associadas a depressão, encontram-se: idade avançada, sexo feminino, condições de saúde, como a incapacidade funcional, doenças crônicas e déficit cognitivo, além das condições socioeconômicas precárias e lares multigeracionais.

Cerca de 6\% de todos os pacientes que realizam consulta médica apresentam critérios para diagnóstico de depressão maior, sendo este número muito maior entre a população idosa, variando de 3 a 15\%. Esta taxa sobe ainda mais se forem analisados os idosos com problemas graves de saúde, chegando a cerca de $40 \%$ entre pacientes com AVC, 35\% entre cancerosos, $25 \%$ entre parkinsonianos, $20 \%$ em portadores de doenças cardiovasculares e 10\% entre diabéticos ${ }^{6}$.

Segundo a Organização Mundial de Saúde, a depressão maior, que foi a quarta causa de incapacitação em 1990 em todo o mundo, será, em 2020, a segunda, perdendo apenas para as doenças do coração ${ }^{7}$.

Conhecer a prevalência de depressão nos idosos da população a ser avaliada neste estudo é de fundamental importância para a saúde pública a fim de que sejam criados mais centros de atenção ao idoso, subsídidos para a realização de ações de promoção à saúde e prevenção de doenças e, principalmente, para que possa se manter o idoso com sua independência funcional e inserido na comunidade. Identificar o perfil do idoso com depressão facilitará a realização de ações individuais e coletivas pelas equipes de Saúde da Família, diminuindo os gastos com atenção secundária e terciária tão saturados atualmente.

\section{Justificativa}

Depressão é altamente prevalente e constitui um grave problema de saúde pública, gerando repercussões sociais e econômicas.

O aumento progressivo da expectativa de vida juntamente com um aumento no número de idosos em relação à população geral gerou a necessidade de se desenvolver uma abordagem diagnóstica e terapêutica específica para a população senil.

Como os transtornos mentais nos gerontes geralmente estão associados a outras patologias, sua abordagem torna-se mais complexa, o que traz importantes consequências para a qualidade de vida e autonomia do idoso. Os 
transtornos mentais mais comuns nesta faixa etária são a depressão, demência, delirium, transtornos da ansiedade e transtornos psicóticos.

É fundamental que o médico da Atenção Primária à Saúde (APS) saiba diferenciar depressão de demência, visto que o tratamento e o prognóstico são bastante diferentes. Aproximadamente 25\% dos pacientes com demência em fase inicial são erroneamente rotulados como deprimidos, e 30\% dos dementes não são reconhecidos como tais ${ }^{8}$. Com isto, vê-se a importância de se aprofundar os estudos nesta área, dada a sua complexidade, além do que, muitas vezes, os sintomas dessas duas doenças coexistem. A depressão é frequente na demência inicial como também pode levar a déficit cognitivo acentuado.

$\mathrm{O}$ interesse por esta pesquisa se deve à pouca quantidade de estudos brasileiros sobre a prevalência de depressão em idosos na APS, sendo extremamente necessário caracterizar o perfil do idoso brasileiro para que se possa desenvolver uma rede de apoio ao idoso de acordo com a nossa realidade.

\section{Objetivos}

\subsection{Objetivo Geral}

Identificar a prevalência de depressão nos idosos da comunidade do bairro Curió no município de Fortaleza

\subsection{Objetivos Específicos}

- Conhecer o perfil socioeconômico e demográfico da população estudada.

- Avaliar os fatores associados a sintomas depressivos no idoso.

- Subsidiar conhecimentos para a realização de ações de promoção à saúde e prevenção de doenças para melhorar a qualidade de vida do idoso atendido nas Unidades de Saúde da Família do município de Fortaleza.

\section{Revisão de literatura}

\subsection{Envelhecimento Populacional Mundial}

$\mathrm{Na}$ Roma Antiga, um indivíduo com 50 anos era considerado idoso. Atualmente, nos países desenvolvidos não é possível falar em velhice antes dos 75 anos. Nos países em desenvolvimento, o ponto de corte para ser considerado idoso é 60 anos. $\mathrm{Na}$ verdade, este serve mais para fins de aposentadoria e estudos demográficos. A tendência é que esses valores sejam aumentados com o progresso da ciência, que, consequentemente, posterga as doenças associadas à velhice para cada vez mais tarde.

Uma população envelhece quando a proporção de velhos aumenta, e esta só aumenta quando a proporção de jovens diminui devido à redução da taxa de fecundidade. A transição demográfica de uma população de jovem para envelhecida deu-se originalmente na Europa, onde a taxa de fecundidade declinou marcadamente, consequência do desenvolvimento social devido à Revolução Industrial, com queda da mortalidade, que levou, a longo prazo, à queda da fecundidade.

As doenças infectocontagiosas, mais prevalentes na população jovem, tendem a reduzir sua incidência, enquanto as doenças crônicas não-transmissíveis (DCNT) aumentam sua prevalência na população idosa, o que caracteriza a mudança no perfil de morbimortalidade que acompanha o processo demográfico, denominado de transição epidemiológica. O grande desafio atualmente é procurar formas de prevenir as DCNT com o objetivo de preservar a autonomia do idoso, fundamental para a manutenção de sua qualidade de vida ao longo dos anos. É fácil compreender a importância dessa meta, visto que um idoso com uma ou mais doenças crônicas controladas é considerado saudável, enquanto um idoso com as mesmas doenças não controladas perde sua capacidade funcional.

\subsection{Envelhecimento Populacional no Brasil}

Os países denominados de Terceiro Mundo, nas últimas décadas, vêm apresentando redução nas taxas de morlidade e, mais recentemente, nas taxas de fecundidade, consequência da melhoria nas condições de vida da popu- 
lação ${ }^{1}$.

A passagem de um país com características de taxas de mortalidade e fecundidade altas para baixas leva a um aumento na expectativa de vida da população e em um aumento no número de pessoas atingindo a senilidade. Em 2025, estima-se que, entre os dez países no mundo com maior número de idosos, cinco serão países em desenvolvimento, incluindo o Brasil no sexto lugar ${ }^{8}$.

$\mathrm{Na}$ época da Segunda Guerra Mundial, a expectativa média de vida era de 39 anos, sendo atualmente de 68. No ano 2000, os idosos representavam $9 \%$ da população brasileira. Este número evolui rapidamente, visto que passou, nos anos de 1960 a 1980, de 3 para 7 milhões de pessoas, sendo de 14 milhões no ano 2000 (aumento de $500 \%$ em 40 anos). Há estimativas de que este número alcançará os 32 milhões em $2020^{4}$.

No Brasil, as principais causas de mortalidade na população idosa são as doenças cardiovasculares, as neoplasias e as doenças do aparelho respiratório. Em relação ao perfil de morbidade dos idosos brasileiros, tem-se que $69 \%$ destes relatam ter pelo menos uma doença crônica, sendo em uma proporção maior entre as mulheres que homens $(75 \%$ e $62 \%$ respectivamente). Dentre as doenças crônicas mais citadas, estão a hipertensão (44\%) e artrite (38\%), seguidos de doenças do coração, diabetes, asma, doença renal crônica, câncer e cirrose?

O processo migratório e a intensa urbanização (em 1940 a população rural era de 68,8\% e em 1980 de 32,4\%) afetaram particularmente a população idosa dos grandes centros ou daqueles que envelheceram nestas cidades, acentuando os problemas de solidão e pobreza nesta faixa etária ${ }^{10}$.

\subsection{Depressão: Fisiopatologia, Diagnóstico e Tratamento}

Segundo Kaplan e Sadock ${ }^{11}$, o humor é um tom emocional persistente, percebido ao longo de uma linha contínua normal de triste e alegre. Os transtornos do humor são caracterizados por sentimentos anormais de depressão ou euforia, com aspectos psicóticos associados em casos mais graves. Os transtornos do humor são divididos em transtornos bipolares - episódios de mania e depressão - e distúrbios depressivos unipolares.

Nos últimos 30 anos, a neuroquímica é a área que vem recebendo maior destaque nas pesquisas sobre a fisiopatologia da depressão. Isso teve seu início a partir do descobrimento da ação dos antidepressivos.

Atualmente, é consenso que a fisiopatologia da depressão é multifatorial, pois nenhuma hipótese até o presente momento explica totalmente o mecanismo da depressão, uma vez que não pode ser atribuída apenas ao hipofuncionamento dos neurotransmissores ou à sua redução nas sinapses.

Estudos recentes com famílias indicam a existência de um componente genético para a depressão. Estima-se que o componente genético represente cerca de $40 \%$ da susceptibilidade para desenvolver depressão unipolar e cerca de $70 \%$ para o transtorno bipolar ${ }^{12}$.

Atualmente, existem sistemas classificatórios que facilitam o diagnóstico de depressão que poderão diminuir a falta de reconhecimento nos serviços de APS desta enfermidade, que chega a 50\% de casos não-diagnosticados. Ajuda ainda a identificar outras formas de apresentação com sintomas mais leves. São eles:

- Distimia: transtorno depressivo crônico com sintomatologia mais leve, presente pelo menos por dois anos com períodos ocasionais e curtos de bem-estar. Além do humor deprimido, o paciente tem de apresentar até três dos seguintes sintomas: diminuição da energia, diminuição da autoconfiança, insônia, dificuldade de se concentrar, choro, diminuição do interesse sexual e outras atividades prazerosas, sentimento de desamparo, inabilidade de lidar com responsabilidade no dia-a-dia, pessimismo com relação ao futuro, retraimento social e diminuição do discurso.

- Transtorno misto de ansiedade e depressão: pacientes com sintomas de ansiedade e depressão sem que nenhum dos dois, considerados separadamente, justifiquem um diagnóstico. Alguns sintomas são autonômicos (tremor, palpitação, boca seca, epigastralgia).

- Depressão subsindrômica: pacientes com sintomas leves 
que não preenchem os critérios, mas apresentam alto risco de apresentarem episódios de depressão futuros.

Para o diagnóstico de depressão, além da história clínica e dos dados obtidos do exame do estado mental, são utilizados sistemas classificatórios como o DSM-IV:

A. Cinco ou mais dos seguintes sintomas estiveram presentes durante o período de duas semanas e representam uma mudança no funcionamento anterior; pelo menos um dos sintomas é (1) humor deprimido ou (2) perda do interesse ou prazer.

1. humor deprimido na maior parte do dia, quase todos os dias, indicado por relato subjetivo ou observação feita por outros;

2. interesse ou prazer acentuadamente reduzido por todas ou quase todas as atividades na maior parte do dia, quase todos os dias;

3. perda ou ganho significativo de peso sem estar de dieta ou diminuição ou aumento do apetite quase todos os dias; 4. insônia ou hipersonia quase todos os dias;

5. agitação ou retardo psicomotor quase todos os dias;

6. fadiga ou perda de energia quase todos os dias;

7. sensação de inutilidade ou culpa excessiva ou inapropriada (que pode ser delirante) quase todos os dias;

8. capacidade diminuída para pensar ou concentrar-se, ou indecisão quase todos os dias;

9. pensamentos de morte recorrentes, ideação suicida recorrente sem um plano específico, tentativa de suicídio ou plano específico para cometer suicídio.

B. Os sintomas não satisfazem os critérios para um episódio misto.

C. Os sintomas causam sofrimento clinicamente significativo ou prejuízo no funcionamento social ou ocupacional ou em outras áreas importantes da vida do indivíduo.

D. Os sintomas não se devem aos efeitos fisiológicos diretos de uma substância ou condição médica geral.

E. Os sintomas não são melhor explicados por luto, isto é, após a perda de um ente querido, os sintomas persistem por mais de dois meses ou são caracterizados por acentuado prejuízo funcional, preocupação mórbida ou desvalia, ideação suicida, sintomas psicóticos ou retardo psicomotor.
A classificação internacional de doenças na sua décima versão (CID-10) apresenta algumas diferenças no diagnóstico de depressão em relação ao DSM-IV. Ela diferencia os episódios depressivos em leve, moderado e grave, podendo ser estes com ou sem sintomas psicóticos.

Os critérios diagnósticos para episódio depressivo segundo o CID-10 são os seguintes:

A) Critérios Gerais para episódio depressivo

1) O episódio deve durar pelo menos duas semanas

2) Não haver sintomas maníacos ou hipomaníacos em qualquer época da vida

3) O episódio não é atribuível ao uso de substâncias psicoativas

B) Critérios principais para episódio depressivo (dois ou mais destes sintomas devem estar presentes sempre)

1) Humor deprimido que definitivamente seja anormal para aquele indivíduo, presente pela maior parte do dia e quase todos os dias, largamente não influenciado pelas circunstâncias e mantido por pelo menos duas semanas.

2) Perda de interesse ou prazer por atividades que normalmente são agradáveis.

3) Energia diminuída ou fadigabilidade aumentada.

C) Sintomas adicionais para episódio depressivo:

1) Perda de confiança ou autoestima.

2) Sentimentos irracionais de autorreprovação ou culpa excessiva ou inapropriada.

3) Pensamento recorrente de morte ou suicídio ou qualquer comportamento suicida.

4) Queixas ou evidências de diminuição da capacidade de pensar ou concentrar-se, tais como indecisão ou vacilação.

5) Alteração na atividade psicomotora com agitação ou lentificação (tanto subjetiva como objetiva).

6) Qualquer tipo de perturbação do sono.

7) Alteração do apetite (diminuição ou aumento), com correspondente alteração do peso.

Pode haver a presença ou ausência de síndrome somática (características melancólicas, neurovegetativas ou físicas):

1) Marcante perda de interesse e prazer em atividades que são normalmente prazerosas. 
2) Falta de reações emocionais a eventos ou atividades que normalmente produzem reação emocional.

3) Levantar-se de manhã duas ou mais horas antes do habitual.

4) Depressão pior pela manhã.

5) Evidência objetiva de retardo ou agitação psicomotora marcante (observada ou relatada por outra pessoa).

6) Marcante perda de apetite.

7) Perda de peso (5\% ou mais do peso corporal no último mês).

8) Marcante perda da libido.

Nos episódios depressivos leves, deve-se encontrar dois ou três sintomas dos mencionados acima, causando angústia ao paciente, porém podendo este seguir com sua rotina diária.

No episódio depressivo moderado, deve-se encontrar quatro ou mais sintomas dos mencionados acima, causando angústia ao paciente e dificultando a vida cotidiana.

No episódio depressivo grave sem sintomas psicóticos, deve-se encontrar vários dos sintomas mencionados os quais são marcantes e causam bastante sofrimento, impedindo o indivíduo de prosseguir com suas atividades diárias.

No episódio depressivo grave com sintomas psicóticos, aparecem vários sintomas mencionados, os quais são acompanhados de sintomas psicóticos, como delírios e alucinações ou mesmo estupor depressivo ${ }^{13}$.

Atualmente, o tratamento da depressão apresenta um leque de opções terapêuticas, permitindo ao médico individualizar o tratamento, levando em consideração aspectos biopsicossociais. Além do tratamento com medicações, tem de se considerar a importância da psicoterapia e mudanças no estilo de vida do indivíduo, envolvendo uma equipe multidisciplinar.

Segundo Souza ${ }^{14}$, os antidepressivos produzem em média uma melhora dos sintomas depressivos de 60 a 70\% no prazo de um mês, enquanto a taxa de placebo gira em torno de 30\%. Essas drogas têm seu uso individualizado para cada paciente, levando-se em consideração aspectos como efeitos adversos, custo, gravidade dos sintomas dro- gas associadas etc. Existem várias classes de antidepressivos como os tricíclicos (amitriptilina, clomipramina, imipramina, maprotilina e nortriptilina), inibidores seletivos da recaptação de serotonina (citalopram, fluoxetina, fluvoxamine, paroxetina e sertralina), inibidores da MAO (moclobemide e tranylcypromine) e outros. As mais usadas e mais eficientes têm sido os tricíclicos e os inibidores da recaptação de serotonina (ISRSs).

Doses inadequadas e não-adesão ao tratamento têm sido as principais causas de falha terapêutica. Os efeitos colaterais mais comuns dos tricíclicos são anticolinérgicos e dos ISRSs são os efeitos sexuais. As drogas devem ser utilizadas para remissão dos sintomas e prevenção de recaídas. Seu início de ação junto à sintomatologia é de cerca de três a quatro semanas. $\mathrm{O}$ tratamento deve ser de pelo menos 16 a 20 semanas, com doses plenas após a melhora ou remissão completa dos sintomas. Em casos graves de depressão com risco de suicídio, características psicóticas e em grávidas, eletroconvulsoterapia deve ser indicada ${ }^{14}$. A hospitalização só deve ser indicada em casos de risco suicida e/ou homicida claro, falta de suporte social ao paciente, abuso de substância grave ou não-cooperação do paciente.

\subsection{Depressão no Idoso: Peculiaridades}

A depressão entre os idosos vistos na APS é bastante comum. Podem se apresentar sob diversas formas como problemas de memória e desordens somáticas inespecíficas como tonturas e distúrbios do sono, o que torna seu diagnóstico mais complicado. A tentativa de tratar estes sintomas isoladamente pode levar à prática da polifarmácia, que compromete a qualidade de vida do geronte. Outra grande dificuldade enfrentada é a ideia de que a maioria dos sintomas apresentados pelos idosos decorre da idade e não de uma patologia.

Dividindo-se os sintomas depressivos em idosos em psicológicos e somáticos, percebe-se que os primeiros são menos prevalentes, contudo mais relevantes para o diagnóstico. Sintomas psicóticos na depressão são mais frequentes em idosos que em adultos, sendo mais comuns os delírios de culpa, persecutórios, de negação, hipocon- 
dríacos e de ciúmes. Sintomas cognitivos também são mais comuns em idosos deprimidos, sendo, às vezes, tão proeminentes, que o quadro é denominado de pseudo-demência. Por isso, é muito importante distinguir o episódio depressivo da demência. Esta se diferencia daquela pelo início abrupto do déficit cognitivo, tendo caráter flutuante entre as consultas e há tendência de se responder "não sei" às perguntas. Pode acontecer de um déficit cognitivo iniciado em um quadro depressivo não remitir totalmente e evoluir para a demência. Contudo, mesmo os pacientes que têm remissão total do déficit cognitivo com o tratamento têm um risco maior de apresentarem demência, chegando a 20\%15.

A etiologia da depressão geriátrica é multifatorial. Podemos citar como fatores os genéticos, que contribuem em menor parte que na depressão de início precoce, fatores metabólicos, devido à diminuição dos neurotransmissores com a idade, a saúde física e o suporte social. As características do quadro depressivo que se inicia na senilidade são: menor frequência de história familiar para transtornos do humor, maior relação com eventos vitais negativos, maior prevalência de demência, maior prejuízo em testes neuropsicológicos e maior número de alterações de exames de neuroimagem estrutural.

Outro fator a ser considerado na depressão geriátrica é a maior incidência do luto. A perda do companheiro tem sido associada a declínio mental e físico, ocorrendo sintomas depressivos cerca de dois a seis meses após a perda. A comorbidade de doenças físicas acentua a incapacidade funcional do idoso. Esta frequentemente associada a doenças cardiovasculares, endócrinas, Parkinson, câncer e dor crônica.

O idoso submete-se obrigatoriamente a estressores psicológicos e sociais como em nenhuma outra faixa etária, encontrando-se em pior situação financeira após aposentadoria, o que resulta em piores condições de moradia e declínio social. A redução da mobilidade e da capacidade de entender o ambiente pela diminuição da acuidade auditiva e visual, além da deterioração da saúde física e mental, coloca o idoso em um grupo pouco privilegiado ${ }^{16}$.

A síndrome depressiva é geralmente associada a patologias clínicas crônicas, e, quando está presente, acaba levando a uma pior evolução e dificuldade para a adesão do paciente ao tratamento, consequentemente com prejuízo da qualidade de vida e maior morbimortalidade como um todo ${ }^{17}$. Como o aparecimento de doenças crônicas é diretamente proporcional ao avançar da idade, isso explica em parte a alta prevalência da depressão na senilidade.

A Escala de Depressão em Geriatria (GDS) é um dos instrumentos mais frequentemente utilizados para a detecção de depressão no idoso. Vem sendo amplamente usada em diversos países, com índices de confiabilidade e validade adequados ${ }^{18}$.

Atualmente, a GDS de 30 questões vem cedendo espaço às versões reduzidas, como a GDS-15, com boa performance na deteç̧ão de novos casos de depressão, sendo esta a escolhida para ser utilizada no presente estudo.

O tratamento da depressão no idoso tem suas particularidades, tendo em vista que o idoso tem mais comorbidades que contraindicam determinados medicamentos, a interação entre eles e até mesmo a diferença no metabolismo corporal em relação ao adulto jovem.

O tratamento requer uma abordagem multiprofissional, ampliando a eficácia da medicação a ser instituída no paciente idoso e potencializando o tratamento farmacológico. Este deve ser iniciado o mais precocemente possível, com o objetivo de reduzir o tempo em que o geronte se torna incapaz. Os antidepressivos são as drogas de escolha para o tratamento da depressão no idoso. Dentre as classes de medicamentos, algumas se mostram melhores que outras com relação a diminuição dos efeitos adversos. Por exemplo, citam-se os inibidores da monoaminoxidase, que apresentam vantagens em comparação aos agentes tricíclicos, por terem menos efeitos anticolinérgicos e não alterarem a condução cardíaca ${ }^{6}$. Por isso, a escolha da medicação deve ser individualizada, respeitando-se as indicações, as contraindicações e seus vários efeitos adversos mais proeminentes em idosos.

A psicoterapia, incluindo as terapias cognitivo-comportamentais, a terapia interpessoal e as terapias de orientação psicodinâmica, deve ser estimulada como parte fundamental do tratamento, pois, na senilidade, o indivíduo, muitas vezes, 
encontra a solidão, o desamparo, a aposentadoria e a perda do cônjuge sem o devido preparo psicológico, perdendo a autoestima e a vontade de viver, necessitando, por isso, de um suporte social para ser reinserido na comunidade em que vive. Cabe, ainda, ao médico do serviço de APS realizar a terapia familiar para resgatar determinados valores esquecidos ao longo dos anos pelos familiares: de respeito e admiração ao idoso pela sua experiência e sabedoria.

\section{Metodologia}

\subsection{Tipo de Pesquisa}

Trata-se de um estudo quantitativo, exploratório e descritivo da prevalência de sintomas depressivos em idosos e sua relação com os dados socioeconômicos e demográficos.

Os dados foram coletados pelos agentes comunitários de saúde (ACS), que enfocou variáveis como estado civil, nível de escolaridade, doenças associadas, renda familiar, autocuidado, dentre outros. Os ACS convidaram todos os idosos da área estudada para responderem ao questionário, entretanto 34 não puderam ou não quiseram participar do processo.

\subsection{Cenário}

A área estudada localiza-se no município de Fortaleza, mais precisamente nas microáreas 1 e 2 da Equipe Saúde da Família de número 66, adscrita na Unidade de Saúde da Família Terezinha Ferreira Parente, localizada no bairro Lagoa Redonda, área metropolitana.

O município de Fortaleza tem, segundo os dados de 2005 fornecidos pelo IBGE, uma população de 2.374.944 habitantes, com extensão territorial de 313,14 Km². Segundo a mesma fonte, a população de idosos é de 160.231 pessoas, sendo 63.098 do sexo masculino e 97.133 do sexo feminino (dados de 2000) ${ }^{4}$.

\subsection{Amostra}

A amostra foi composta de 85 idosos que aceitaram participar do estudo. De acordo com os dados do Sistema de Informação da Atenção Básica (SIAB), juntamente com os dados do consolidado anual por micro-área do ano de
2005, o número de famílias dessas microáreas era de 587 famílias, que é uma população estimada de 2.428 habitantes. O número de idosos dessas microáreas, segundo o levantamento dos agentes comunitários de saúde, é de 119 idosos, o que corresponde a 4,9\% do total de pessoas desta área estudada. A proporção entre os sexos feminino e masculino nesta faixa etária é de 55,4\% e 44,6\% respectivamente.

\subsection{Instrumento de coleta de dados}

Utilizou-se a Escala de Depressão Geriátrica (GDS) abreviada de Yesavage, com 15 itens, e um questionário estruturado, que avalia variáveis que podem estar associadas com a depressão, como nível de escolaridade, estado civil, renda familiar, moradia em domicílios multigeracionais, dentre outras respondidas mediante entrevista com todos os idosos.

A escala de Depressão Geriátrica é um dos instrumentos mais frequentemente utilizados para a detecção de sintomas depressivos no idoso. Almeida e Almeida ${ }^{19}$ demonstraram que as versões brasileiras da GDS 15 e GDS 10 oferecem medidas válidas para o diagnóstico de episódio depressivo maior de acordo com os critérios da CID-10 e DSM-IV. O ponto de corte 5/6 (não caso/caso) para a GDS 15 produziu índices de sensibilidade de 85,4\% e especificidade de 73,9\% para o diagnóstico de episódio depressivo maior de acordo com a CID-10. Segundo Paradela et al. ${ }^{33}$, a Escala GDS-15 pode ser uma escala de grande utilidade no rastreamento de indivíduos idosos, suspeitos de transtorno depressivo em ambientes não-especializados, sendo a escala bem aceita pelos usuários, a metodologia aplicada simples, eficiente e de baixo custo, podendo ser replicada em ambientes como hospitais, domicílios, programas de saúde da família e em casas geriátricas de longa permanência.

Os ACS receberam treinamento para a realização da aplicação do questionário estruturado e da Escala de Depressão Geriátrica, tendo inclusive recebido supervisão em seus primeiros questionários para clareamento de dúvidas que surgiram na aplicação dos mesmos. 


\subsection{Análise dos dados}

Os dados coletados através da aplicação da Escala de Depressão Geriátrica e questionário estruturado foram expressos em gráficos, tabelas e quadros elaborados pelo Programa Epi Info versão 6.04d para análise e interpretação.

\section{Resultados}

Os resultados foram analisados, considerando-se os critérios de não-caso e caso de depressão segundo a Escala de Depressão Geriátrica - EDG, isto é, os que apresentaram valor igual e inferior a cinco foram considerados não-caso, e os casos eram os que possuíam valor igual e superior a seis ${ }^{19}$.

Do total de 85 idosos entrevistados, as mulheres apresentaram-se em maior número, perfazendo um total de $67,1 \%$ da amostra total, sendo os homens $32,9 \%$. Analisando ainda o gênero quanto a ser ou não caso, pode-se observar que as maiores proporções eram de mulheres com 84,4\% e $56,6 \%$ para caso e não-caso, respectivamente. Quanto à razão de prevalência verificou-se nas mulheres ser maior 2,65 vezes que nos homens, com IC 95\%: 1,14- 6,15 e p< 0,08.

A prevalência de depressão na amostra estudada correspondeu a 37,6\% (32 pessoas), sendo encontrada uma grande diferença entre os sexos feminino e masculino, com $84,4 \%$ e $15,6 \%$, respectivamente.

$\mathrm{Na}$ distribuição dos idosos acompanhados no estudo por faixa etária observou-se maiores proporções na faixa de 65 a 69 anos, seguida dos grupos etários de 60 a 64 anos e 75 a 79 anos, respectivamente com 32,9\%, 23,5\% e $16,5 \%$. Analisando ainda estes dados correlacionados com os casos e não-casos de depressão geriátrica, foram verificados que não houve diferença significativa entre aqueles que eram caso e os não-casos com $p<0,275$. As maiores proporções nos casos se encontravam nas faixas de 65 a 69 anos, com 37,5\%; de 60 a 64 anos e de 70 a 74 anos com mesma proporção de 21,9\%. (Figura 1).

Analisando os idosos do estudo quanto a serem ou não alfabetizados, é possível observar que não houve diferenças estatísticas entre ser ou não caso com depressão geriátrica. As proporções encontradas foram de $40 \%$ de alfabetizados e $60 \%$ de não-alfabetizados, nos casos, e, den- tre os não-casos, pôde-se observar 64,7\% para alfabetizados e $58,5 \%$ para os não-alfabetizados.

Em relação ao estado conjugal, as maiores proporções registraram-se nos casados, tanto entre os casos quanto os não-casos, com 53,1\% e 56\%, respectivamente. Mesmo com estes dados, não foi verificada significância estatística. Nos idosos sem depressão do estudo observou-se uma proporção de $32 \%$ entre viúvos e separados.

Quanto à religião, observou-se que a católica representou $71,9 \%$ e $75,5 \%$ para casos e não-casos, respectivamente.

A frequência de casos de depressão segundo a Escala de Depressão Geriátrica foi maior nas pessoas da amostra total que tinham origem urbana, com um total de 54,8\% dos casos contra 45,4\% de origem rural. Este estudo não analisou o tempo decorrido da migração dos idosos de origem rural.

Analisando-se a renda familiar, em salários-mínimos (SM), foi verificado que $74,1 \%$ dos idosos acompanhados apresentavam renda de 1 a 2 SM, 18,8\% e 7,1\% para menos de 1 e acima de 2 SM, respectivamente. Quando comparados casos e não-casos, observou-se a mesma sequência, isto é, maiores proporções na renda de 1 a $2 \mathrm{SM}$, seguida de menos de 1 e acima de 2, com as proporções de 68,8\%, 21,9\% e $9,4 \%$ para os casos e $77,4 \%, 17,0 \%$ e $5,7 \%$ nos não-casos.

No presente estudo, verificou-se que $74,2 \%$ dos casos de depressão detectados nos idosos da amostra total moram em domicílios multigeracionais. (Figura 2).

Dos 32 casos de depressão detectados pela Escala de Depressão Geriátrica, 20 (62,5\%) apresentam hipertensão arterial sistêmica (HAS) e/ou diabetes mellitus (DM).

$10 \%$ apresentam diabetes mellitus, 55\% apresentam hipertensão arterial sistêmica e 35\% apresentam ambas as doenças. (Figura 3 e Tabela 1).

Avaliando-se outras comorbidades (autorreferidas), os problemas mais comumente relatados foram: catarata (10 pessoas), distúrbios do sono (14 pessoas) e dores na coluna (15 pessoas).

Avaliando-se a capacidade funcional dos idosos da amostra estudada, verificou-se que oito idosos do estudo 
tinham comprometimento de alguma atividade básica de vida diária (AVDs), dos quais um não se higienizava sozinho e sete não andavam sozinhos.

$\mathrm{Na}$ amostra estudada, não houve diferença na frequência de casos e não-casos de depressão em pessoas que estiveram internadas nos seis meses anteriores à aplicação do questionário. Também não houve correlação entre casos de depressão e dependência de álcool.

A proporção de idosos tabagistas encontrada no estudo foi de $26,5 \%$, dentre estes, observou-se que $45,5 \%$ foram registrados nos casos de depressão segundo a Escala de Depressão Geriátrica contra $54,5 \%$ de fumantes nos não-casos.

\section{Discussão e considerações finais}

O estudo realizado se mostrou bastante relevante para o conhecimento da área de abrangência da Equipe
Rosa do Centro de Saúde da Família Terezinha Parente, visto que 85 idosos participaram do mesmo, propiciando uma série de dados interessantes para a realização de um trabalho mais aprofundado na APS dentro da esfera da Saúde Mental.

O presente estudo se propôs a identificar a prevalência de depressão nos idosos da área de abrangência de uma Equipe Saúde da Família localizada no bairro Curió no município de Fortaleza. Com esses dados, procurou-se correlacionar a presença de sintomas depressivos com fatores socioeconômicos, com o objetivo de compreender a dinâmica da doença e traçar ações para melhorar a qualidade de vida dos idosos com depressão. Utilizou-se a Escala de Depressão Geriátrica com 15 questões (GDS-15), que oferece medidas válidas para o diagnóstico de episódio depressivo maior de acordo com os critérios da CID-10 e DSM-IV. O ponto de corte 5/6 (não-caso/caso) para a GDS-15 pro-

Figura 1. Proporção de idosos acompanhados no estudo segundo Escala de Depressão Geriátrica (EDG) e faixas etárias.

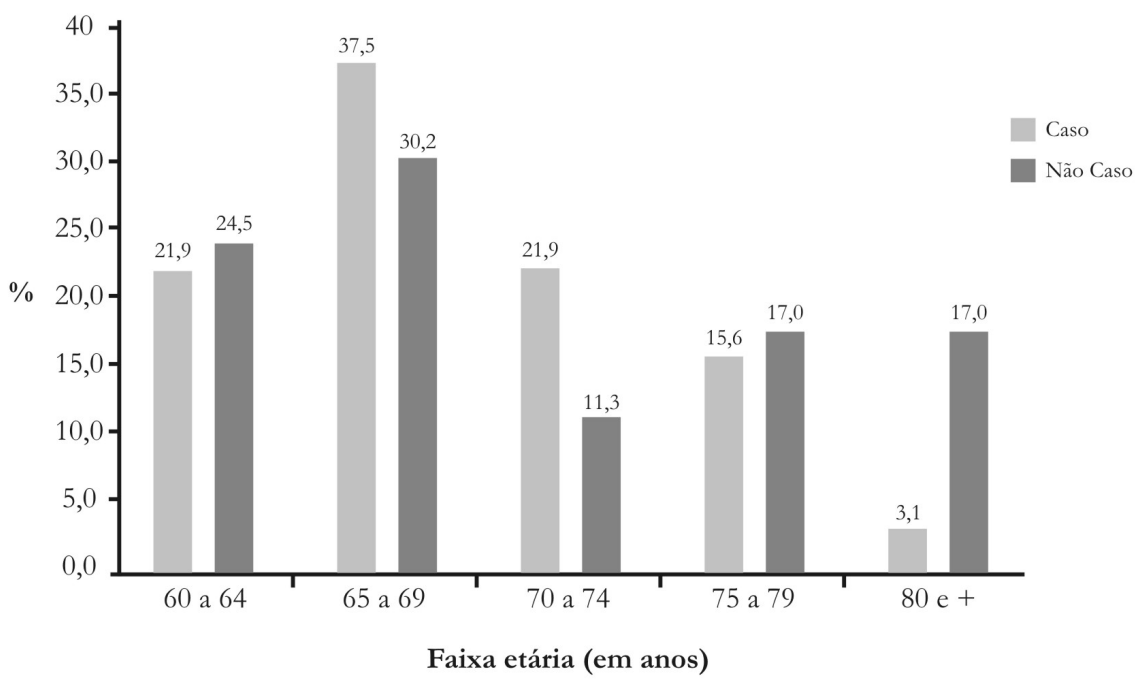


Figura 2. Proporção de idosos acompanhados no estudo segundo Escala de Depressão Geriátrica (EDG) e domicílios multigeracionais.

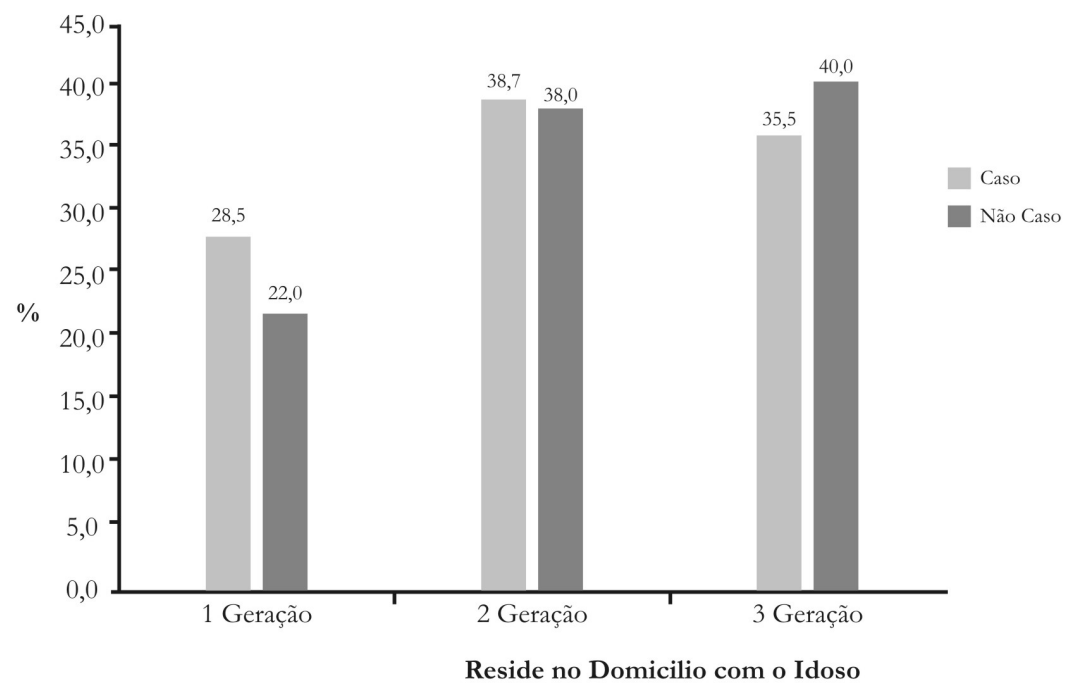

Figura 3. Proporção de idosos acompanhados no estudo segundo a Escala de Depressão Geriátrica e presença de HAS e/ou DM.

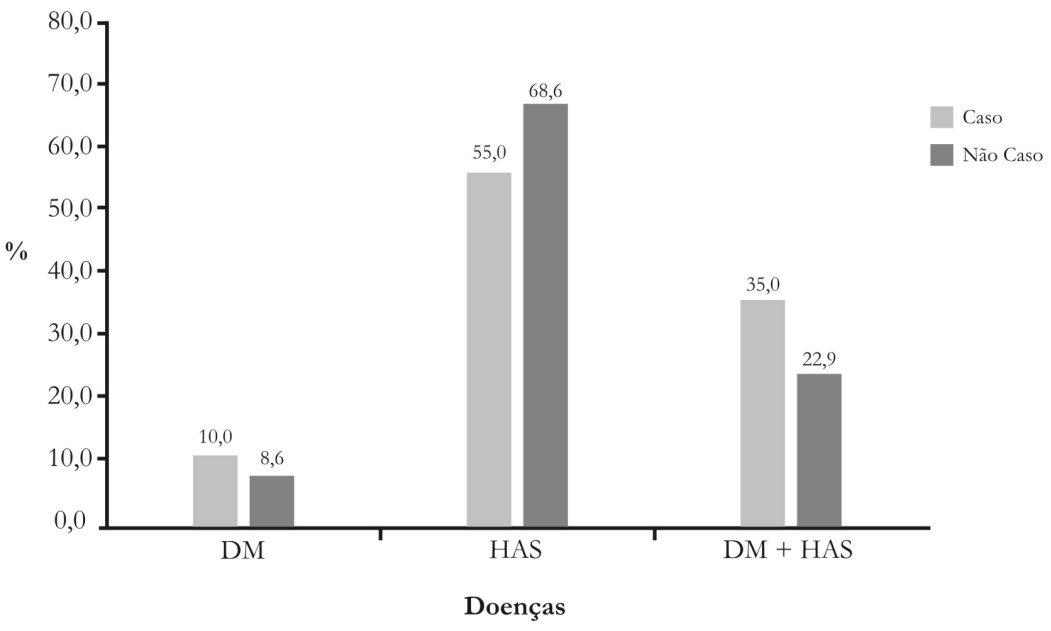

Tabela 1. Número e proporção de idosos acompanhados no estudo segundo Escala de Depressão Geriátrica e doenças associadas.

\begin{tabular}{lcccccc}
\hline $\begin{array}{c}\text { Número de proporção de idosos acompanhados no estudo segundo } \\
\text { Escala de Depressão Geriátrica e doenças associadas }\end{array}$ \\
\hline & \begin{tabular}{c} 
Caso \\
\cline { 2 - 6 }
\end{tabular} & Não Caso & $\mathrm{X}^{2}$ & $\mathrm{P}$ \\
\cline { 2 - 6 } Doenças Associadas & 2 & 10.0 & 3 & 8.6 & & \\
DM & 11 & 55.0 & 24 & 68.6 & 1.09 & $\mathrm{P}<0.581$ \\
HAS & 7 & 35.0 & 8 & 22.9 & & \\
DM + HAS & & & & & & \\
\end{tabular}


duziu índices de sensibilidade de $85,4 \%$ e especificidade de $73,9 \%$ para o diagnóstico de episódio depressivo maior segundo o CID-10 19 .

O processo de envelhecimento populacional em curso no país tem aumentado a frequência de doenças psiquiátricas, entre as quais a depressão, que é a desordem mais comum nos idosos. As taxas de prevalência variam de 5 a 35\%, considerando as diferentes formas e a gravidade da depressão ${ }^{20}$.

A prevalência de depressão no presente estudo foi de $37,6 \%$, percentual maior do que a prevalência geralmente encontrada em outros estudos. Em idosos vivendo na comunidade, essa prevalência situa-se entre 2 a 14\% e em idosos que residem em instituições, a prevalência da depressão chega a $30 \%{ }^{21}$. A prevalência de depressão no idoso em comunidade tem sido alvo de discussões em virtude de resultados díspares nos estudos, com taxas variando de 5 a 35\% ${ }^{22}$. Em um serviço de emergência em saúde mental de São Paulo, depressão foi o diagnóstico mais frequente em $41,9 \%$ dos pacientes idosos atendidos ${ }^{23}$. A diferença na prevalência encontrada na comunidade do Curió pode se dever ao fato de que a área de abrangência do estudo corresponde a uma área de risco 1, que é definida pela Secretaria de Saúde do município de Fortaleza, por ser um território onde a população residente tem maiores necessidades por riscos ambientais, sanitários e habitacionais, sem infraestrutura básica para habitação sendo áreas cadastradas pela Defesa Civil.

A proporção entre os sexos no estudo foi de $67,1 \%$ para mulheres e 32,9\% para homens. No Brasil, como em outros países do mundo, há um maior número de mulheres na faixa etária idosa (55\%), diferença esta que se acentua com o avançar da idade ${ }^{24}$. A distribuição de casos entre os sexos foi de $84,4 \%$ para mulheres e $15,6 \%$ para homens na população estudada, diferença esta que pode ser explicada pela maior expectativa de vida das mulheres em relação aos homens. Este dado correlaciona-se com outros estudos, sendo um dado bastante conhecido na geriatria ${ }^{25}$. Nos percentuais relativos ao sexo, no grupo de deprimidos, encontrou-se a maioria de mulheres em relação aos homens em estudo epidemiológico em participantes do Programa Universidade Aberta à Terceira Idade ${ }^{20}$. Fatores socioculturais associados a experiências adversas e fatores psicológicos associados com maior vulnerabilidade a eventos estressores também contribuem para esta diferença entre os sexos feminino e masculino ${ }^{26}$.

A prevalência de depressão foi maior nos idosos mais jovens, com 59,38\% dos casos entre 60 e 69 anos, $37,5 \%$ entre 70 e 79 anos e $3,12 \%$ acima de 80 anos, dado semelhante a alguns estudos de prevalência de depressão em idosos que afirmam que, quanto menor a idade, maior a média de sintomas depressivos. Em estudo realizado em Unidades Primárias de Saúde no município de Fortaleza, $59,5 \%$ pertenciam à faixa etária de 60 a 69 anos de idade e $40,4 \%$ à faixa de 70 a 79 anos de idade, dado semelhante ao encontrado em nosso estudo ${ }^{25}$. Outro inquérito afirma que a taxa de depressão no idoso predomina na faixa de 55 a 64 anos, com um pico entre 60 e 69 anos $^{27}$.

Em relação à situação financeira dos idosos do presente estudo, verificou-se que apenas 9,4\% dos idosos com depressão viviam com renda superior a dois saláriosmínimos, o que mostra a alta taxa de pessoas acima de 60 anos que não possuem trabalho remunerado, mostrando a correlação desfavorável entre a pobreza e depressão, presente em outro estudo populacional ${ }^{28}$.

Um importante dado do presente estudo, mesmo não apresentando significância estatística, foi a alta proporção de pessoas não-alfabetizadas que tinham depressão segundo a Escala de Depressão Geriátrica com 60\% dos casos, mostrando a correlação entre menor escolaridade e maior número de idosos deprimidos encontrada em outros estudos $^{29}$. A associação encontrada entre escolaridade e depressão, observada na menor escolaridade/maior número de idosos deprimidos também foi demonstrada no estudo de Cacciatore $^{20}$. Assim como para outras doenças de caráter crônico como HAS e DM, a maior escolaridade mostrou-se como fator protetor importante para a ocorrência de sintomas depressivos ${ }^{28}$.

A prevalência de idosos sem depressão foi maior nos casados que nos viúvos ou separados, mostrando que 
a solidão ou luto pela morte de um cônjuge na senilidade pode ser fator de risco para desenvolvimento de sintomas depressivos ${ }^{25}$.

Observou-se que $74,2 \%$ dos casos de depressão detectados nos idosos da amostra total moram em domicílios multigeracionais, ou seja, com filhos e netos, o qual representa uma parcela da população de idosos que tende a ser mais pobre, com mais problemas de saúde e mais dependente no dia-a-dia. Isto mostra a relação entre a situação social desfavorável e os sintomas depressivos apresentados pelos entrevistados. Apenas quatro idosos moravam sozinhos, ou seja, a grande maioria vivia com familiares, caracterizando os arranjos familiares multigeracionais típicos de segmentos socioeconômicos mais desfavorecidos ${ }^{30}$.

Como já dito anteriormente, dentre as pessoas com 60 anos e mais, com alguma das duas doenças citadas, que são casos de depressão segundo a Escala de Depressão Geriátrica, 10\% apresentam diabetes mellitus, 55\% apresentam hipertensão arterial sistêmica e 35\% apresentam ambas as doenças.

A hipertensão arterial sistêmica parece ocorrer mais em pacientes com sintomas depressivos e ansiosos, e é clara a correlação entre diabetes mellitus e depressão, em que a prevalência gira em torno de $11 \%$ a $31 \%$, enquanto a presença de diabetes aumenta em duas vezes o risco de depressão ${ }^{17}$.

Avaliando-se outras comorbidades (autorreferidas) nos idosos da amostra total, verificou-se que dentre os casos de depressão segundo a Escala de Depressão geriátrica, os problemas mais comumente relatados foram: catarata (10 pessoas), distúrbios do sono (14 pessoas) e dores na coluna (15 pessoas), sendo este último o sintoma autorreferido em maior número de pessoas. O Inquérito Canadense de Saúde Pública que estudou 118.533 idosos revelou que a lombalgia crônica foi um importante preditor de depressão para idosos ${ }^{31}$.

Dos oito idosos que apresentavam alguma AVD comprometida, seis estão entre os casos de depressão segundo a Escala de Depressão Geriátrica, demonstrando a correlação, nesta população, entre algum grau de dependência no dia-a-dia e sintomas depressivos.

No presente estudo, não houve associação entre tabagismo e depressão, visto que dos idosos tabagistas, 45,5\% foram registrados nos casos de depressão segundo a Escala de Depressão Geriátrica contra 54,5\% de fumantes nos não-casos. Este dado vai de encontro com outros estudos como o de Gazalle et al. ${ }^{28}$, que mostrou associação entre tabagismo e sintomas depressivos. Outro estudo afirmou a associação bem estabelecida entre dependência ao tabaco e diversas formas de transtornos ansiosos e depressivos $^{28}$.

\section{Recomendações}

A Saúde da Família pode ser uma estratégia eficiente para fazer face ao desafio de incluir efetivamente a Saúde do Idoso na APS, mas o cuidado ao idoso deve ser incorporado ao programa de forma específica, incluindo previsão de financiamento das ações e estabelecimento de uma rede de suporte institucional ${ }^{32}$.

Com base no estudo realizado nos idosos da Equipe de Saúde da Família do Bairro Curió, pode-se propor várias ações de promoção de saúde e prevenção de doenças para melhorar a qualidade de vida do idoso atendido nas Unidades Básicas de Saúde da Família do Município de Fortaleza, fator este de suma importância no aparecimento de sintomas depressivos:

- Assegurar efetivamente os direitos inerentes aos idosos presentes no Estatuto do Idoso pela lei $\mathrm{N}^{\circ} 10.741$, de $1^{\circ}$ de outubro de 2003.

- Criar centros de convivência para os idosos por regionais para que eles tenham atendimento multidisciplinar inclusive com suporte social, jurídico, cultural e pedagógico.

- Realizar treinamento específico das equipes de Saúde da Família para realizar atendimento global dos idosos das áreas adscritas.

- Informar sistematicamente a comunidade geral sobre a importância do idoso na sociedade, procurando inserirlhes na mesma de acordo com as particularidades de cada população.

- Realizar parceiras entre as secretarias municipais no sentido 
de otimizar a solução de obstáculos presentes na comunidade que são relacionados a fatores vitais para o aparecimento de depressão nesta faixa etária (incluir as secretarias de educação, infraestrutura, saúde, segurança e ação social). - Estimular as empresas particulares com incentivos fiscais a aproveitar a população idosa como mão-de-obra em diversos setores para melhoria financeira dos mesmos e ao mesmo tempo para melhorar a autoestima dos idosos, que, muitas vezes, sentem-se "inúteis" devido à sua idade.

- Procurar incentivar nas escolas o respeito ao idoso, garantindo aos idosos dignidade e cidadania.

- Realizar capacitação dos cuidadores informais, recebendo orientações direcionadas para prestar um cuidado adequado ao idoso, incluindo medidas preventivas para evitar a dependência precoce.

Captar recursos da própria comunidade para melhorar a qualidade de vida do idoso dentro de seu ambiente familiar, realizando parcerias com centros comunitários, igrejas, associações do bairro e escolas.

\section{Referências}

1. Kalache A, Veras RP, Ramos LR.. O envelhecimento da população mundial: um desafio novo. Rev. Saúde Pública. 1987 jun.; 21(3): 200-210.

2. Ramos LR, Veras RP, Kalache A. Envelhecimento populacional: uma realidade brasileira. Rev. Saúde Pública. 1987 jun; 21(3): 211-224.

3. Santana RLF, Pouchain GC, Bissi LF. A Previdência Social e o Censo 2000: perfil dos Idosos. Informe da Previdência Social. 2002 set.; 14(9). Disponível em: <http:// 66.102.1.104/scholar?hl=pt-BR\&lr=\&q= cache:V7 onWnSRdQQJ:www.previdenciasocial.gov.br/docs / inf_setembro02.pdf+>. Acesso em: 25 junho 2006.

4. Instituto Brasileiro de Geografia e Estatística. Contagem populacional e projeções demográficas preliminares; 2003. Disponível em: < http://www.datasus.gov.br> Acesso em: 25 de jun de 2006.

5.Mittelmark MB. The epidemiology of aging. In Hazzard, W.R. et al. Principles of geriatric medicine and gerontology. 3 ed. New York: Mc Graw-Hill; 1994. p.135-51.
6. Adelman AM, DMP. 20 Problemas + Comuns Geriatria. Rio de Janeiro: Revinter; 2004. 392p.

7. World Health Organization. Population aging: a public health challenge. Geneva: World Health Organization Press Office, 1998.

8. Freitas EV et al. Tratado de Geriatria e Gerontologia. Rio de Janeiro: Guanabara Koogan; 2002. 1187p.

9. Rouquayrol MZ, Almeida Filho N. Epidemiologia \& Saúde. 6 ed. Rio de Janeiro: Medsi; 2003. 708p.

10. Veras RP et al. Crescimento da População Idosa no Brasil: transformações e conseqüências na sociedade. Rev. Saúde Pública. 1987; 21: 225-33.

11. Kaplan HI, Sadock BJ. Manual de Psiquiatria Clínica. 2 ed. Porto Alegre (RS): Artmed, 1998; 444p.

12. Lafer B, Valladão Filho HP. Genética e fisiopatologia dos transtornos depressivos. Rev. Bras. Psiquiatr. 1999 maio; 21 (supl.1): 12-17.

13. Grevet EH, Knijnik L. Diagnóstico de depressão maior e distimia. Rev. AMRIGS. 2001; 45 (3-4): 108-110.

14. Souza FGM. Tratamento da depressão. Rev. Bras. Psiquiatr. 1999 maio; 21(supl.1): 18-23.

15. Carvalho Filho ETC, Netto MP. Geriatria: Fundamentos, clínica e terapêutica. 2 ed. São Paulo: Atheneu; 2006. p. 155-170.

16. Forlenza OV. Etiopatogenia da depressão no idoso. Rev. Psiquiatria Clínica. 1994; 21 (3): 87-94.

17. Teng CT, Humes EC, Demetrio FN. Depressão e comorbidades clínicas. Rev. Psiquiatr. Clín. 2005 maio-jun; 32 (3): 149-159.

18. Montorio I, Izal M. The Geriatric Depression Rating Scale: a review of its development and utility. Int Psychoageriatr. 1996; 8:103-112.

19. Almeida OP, Almeida SA. Confiabilidade da versão brasileira da Escala de Depressão em Geriatria (GDS) versão reduzida. Arq. Neuro-Psiquiatr. 1999 jun; 57(2B): 421-426.

20. Leite VMM et al. Depressão e envelhecimento: estudo nos participantes do Programa Universidade Aberta à Terceira Idade. Rev. Bras. Saude Mater. Infant. 2006 janmar; 6(1): 31-38. 
21. Stella F, Gobbi S, Corazza DI, Costa JLR. Depressão no Idoso: diagnóstico, tratamento e benefícios da atividade física. Motriz. 2002 Set-Dez; 8(3): 91-98.

22. Rocha FL. Depressão no idoso. J. Bras. Psiquiatria. 1993; 42 (9): 483-90.

23. Almeida OP. Idosos atendidos em serviço de emergência de saúde mental: características demográficas e clínicas. Rev. Bras. Psiquiatr. 1999 jan.-mar; 21(1): 12-18.

24. Garrido R, Menezes PR. Brazil is aging: good and bad news from an epidemiological perspective. Rev. Bras. Psiquiatr. 2007 abr.; 24 (supl.1): 3-6.

25. Castelo MS. Validade da Escala de depressão geriátrica em unidades primárias de saúde na cidade de Fortaleza, Ceará. UFC, 2004. 125p. [Dissertação]. Faculdade de Medicina, Universidade Federal do Ceará, 2004.

26. Gazalle FK et al. Sintomas depressivos e fatores associados em população idosa no Sul do Brasil. Rev. Saúde Pública. 2004 jun.; 38(3): 365-371.

27. Grinberg LP. Depressão em idosos - desafios no diagnóstico e tratamento. Rev. Bras. Med. 2006; 63 (7): 317 330.

28. Gazalle FK, Hallal PC, Lima MS. Depressão na população idosa: os médicos estão investigando? Rev. Bras. Psiquiatr. [online]. set. 2004; 26(3): 145-149. [citado 25 Junho 2006]. Disponível em: <http://www.scielo.br/ scielo.php? script $=$ sci_arttext\&pid $=\mathrm{S} 1516-4446$ 2004000300003\&lng=pt\&nrm=iso $>$.

29. Pinto JLG et al. Características do Apoio Social oferecido a idosos de área rural assistida pelo PSF. Ciência e Sáude Coletiva. 2006 jul-set; 11 (3): 753-764.

30. Coelho Filho JM, Ramos LR. Epidemiologia do Envelhecimento no Nordeste do Brasil: resultados de inquérito domiciliar. Rev Saúde Pública. 1999; 33(5): 445-453.

31. Canineu PR. Depressão no idoso In: Netto MP (Org). Tratado de Gerontologia. Atheneu; 2007. p. 293-300.

32. Caldas CP. Envelhecimento com dependência: responsabilidades e demandas da família. Cad. Saúde Pública. 2003 maio-jun; 19 (3):773-781.

33. Paradela EMP, Lourenco RA, Veras RP. Validação da escala de depressão geriátrica em um ambulatório geral.
Rev. Saúde Pública. 2005 dez; 39(6): 918-923.

\section{Endereço para correspondência:}

Rua do Corrente, 333, Casa 5

Edson Queiroz

Fortaleza - CE

CEP: 60812-290

\section{Endereço eletrônico:}

carinabezerra@oi.com.br 